\title{
Experience with Silicon Detectors at the DELPHI Experiment, LEP
}

\author{
P. Collins \\ CERN, CH-1211 Geneva 23, Switzerland
}

\begin{abstract}
New measurements are reported on modules of both the current DELPHI vertex detector, which was installed in 1994, and the upgraded detector which will be installed in 1996. These measurements include an analysis of the phenomenon of "micro-discharge' noise on the ohmic side of double sided detectors, an observation of variable depletion regions on double sided detectors, and the first test results for the $24 \mathrm{~cm}$ long back-to-back modules for the upgraded detector. For the detector presently in operation it has been possible to confirm measurements with tracks from the DELPHI data.
\end{abstract}

Proceedings presented at the 2nd International Symposium on Development and Application of Semiconductor Tracking Detectors, Hiroshima, Oct. 10 -13, 1995. 


\section{Introduction}

The current DELPHI silicon microvertex detector will be upgraded in 1996 to give an increased angular coverage in theta. In this paper new measurements made on modules from both the current and the future detector are described. These include an analysis of the position dependent efficiency of the ohmic side strips on a double sided module and a measurement of 'micro-discharge' noise. For the upgraded detector, test-bench measurements of the new modules are described.

\section{The 1994 detector}

The current DELPHI silicon strip microvertex detector was installed in the experiment in early 1994 and has contributed to the reconstruction and analysis of around 2 million $Z^{0}$ decays. It consists of three layers, termed Closer, Inner, and Outer, which lie at average radii of $6.3,9.0$ and $10.9 \mathrm{~cm}$ respectively. Each layer consists of 24 overlapping modules containing 4 silicon detectors. Of these, the Closer and Outer layers contain double sided detectors, and the Inner provides a single sided readout. The lowest angle covered in the $\theta$ direction varies from $25^{\circ}$ for the Closer layer to $45^{\circ}$ for the Outer layer. A full description of the detector and its performance may be found in [1]. In this section we discuss the Closer layer, which will be reinstalled unchanged in the upgraded detector in 1996, and describe some new measurements carried out on this layer.

\subsection{The Double Sided Closer Layer}

Each of the 24 modules in the double sided Closer layer consists of two electrically independent pairs of silicon detectors. The innermost detectors are $7.9 \mathrm{~cm}$ long, and the the outermost detectors are $6 \mathrm{~cm}$ long. The diodes on the $p$ side of the detectors are parallel to the beam axis and provide a measurement in the $\mathrm{R} \phi$, or transverse, direction, and the $n$ side diodes are orthogonal to the beam axis and provide a measurement in the $\mathrm{Rz}$, or longitudinal, direction. The signals from the $\mathrm{Rz}$ side are carried to the readout electronics by means of a second layer of metal strips, orthogonally oriented to the diodes, and separated from the first layer of metal strips by about $5 \mu \mathrm{m}$ of polyimide. With this arrangement there is negligible extra material in the sensitive region of the detector. The $\mathrm{Rz}$ signals are multiplexed, with three compartments of varying pitch on the innermost detectors and a single compartment of constant pitch on the outermost detectors. A full description may be found in [1].

\subsubsection{Ohmic Side Strip Separation}

Due to the electron accumulation layer at the $\mathrm{Si}-\mathrm{SiO}_{2}$ interface it is necessary to isolate the $n$ side strips from each other. In the Closer layer the technique used is that of field plates over the strips. The metal readout line is extended over the edge of the diode by $4-5 \mu \mathrm{m}$ to create a field which repels the electrons. As the detector is depleted, the field plate voltage becomes different to that of the $n$ strips. At a voltage difference of more than $10 \mathrm{~V}$ the strip separations exceed $100 \mathrm{M} \Omega$, which is sufficient for charge collection. If the depletion zone does not extend to the $n$ side the strips will not be separated. The response of the ohmic side was investigated both with the DELPHI data themselves, and through 
laser tests in the laboratory. It was found that for certain depletion voltages the response of the detector was non uniform, with small regions of inefficiency distributed randomly on the detector. As the depletion voltage was increased the inefficient regions became smaller and at a certain voltage were found to vanish completely. This phenomenon probably relates to an inhomogeneity in the silicon, resulting in varying depletion characteristics at certain points. The voltage at which the detector is $100 \%$ efficient varies from plaquette to plaquette. In figure 1 we show the response of the ohmic side of one $7.9 \mathrm{~cm}$ detector to a laser pulse. The laser is shone on the $p$ side of the detector, so the charge collected drifts through the silicon and surface effects are avoided. At the four different depletion voltages of $60 \mathrm{~V}, 75 \mathrm{~V}, 80 \mathrm{~V}$ and $85 \mathrm{~V}$, the detector shows an efficiency for the ohmic side strips of $55 \%, 93 \%, 95 \%$ and $97 \%$.

\subsubsection{A Measurement of Micro-discharge Noise}

Due to the biasing scheme chosen for the detectors, the readout lines on the $p$ and $n$ sides lie at the same potential. It is therefore possible to bond the detectors with one 'flipped' with respect to the next, so that $p$ and $n$ side signals are read out together. This arrangement optimises the signal over noise $(\mathrm{S} / \mathrm{N})$ by balancing the capacitive load on the input amplifiers, so that the more noisy $n$ strips are always paired with $p$ strips. In the Closer layer, due to the different lengths of the two detectors, the $\mathrm{S} / \mathrm{N}$ is still slightly different on the two sides. For a m.i.p. traversing the detector the measured $\mathrm{S} / \mathrm{N}$ is 13 on the side corresponding to the $p$ side of the $7.9 \mathrm{~cm}$ detector, and 11 on the opposite side. This amount of noise is lower than it would be for two $n$ sides joined together, but still somewhat higher than the level that would be expected, given the total capacitative load of the detectors.

The noise seen in the Closer layer was investigated to see if its origin could be linked to so called 'micro-discharge' or 'popcorn' noise. This type of noise, arising from the strong fringe fields which can be created at the edges of the coupling capacitors, has been extensively described [2]. It develops at a characteristic 'turn-on' voltage, and then quickly rises with increasing voltage. Above this voltage it is strongly inversely correlated to temperature. The effect is fully reversible and reproducible. In principle the DELPHI Closer layer is particularly susceptible to this kind of noise, as the structure of the field plates, where the aluminium lines extend beyond the diode, coupled to the fact that the full biasing voltage is sustained over the coupling capacitor, means that a high field is expected to develop around the edge of the strips. To test for the effect the noise in the detector was measured at a range of voltages and temperatures. The detector was cooled with $\mathrm{N}_{2}$ and the temperature monitored using a probe mounted on the detector with heat conducting paste. The result is shown in figure 2. It can be seen that in the operating regime of DELPHI the noise is constant with varying temperature. However, above approximately $90 \mathrm{~V}$ the noise starts to increase rapidly, showing the largest values at low temperature, which is indicative of micro-discharge noise. In the inset the leakage current, which is also expected to show a jump at the 'turn-on' voltage for micro-discharge noise, is plotted. This measurement was only made at a temperature of $-10^{\circ} \mathrm{C}$, as the leakage current was measured as a total of guard and bulk, and at room temperature it is dominated by guard ring current. This study shows that the detectors are operated in DELPHI in a stable regime below the onset of micro-discharge noise. 


\section{The 1996 upgrade}

In $1996 \mathrm{LEP}$ will raise the energy of $e^{+} e^{-}$collisions to $\sqrt{ } s=161 \mathrm{GeV}$, the threshold energy for $W^{+} W^{-}$production. The energy will later be increased still further, to $\sqrt{ } s=180$ $\mathrm{GeV}$, in late 1996/1997. Important physics objectives at these high energies include the measurement of the $W$ mass, and the search for new particles such as the Higgs boson and supersymmetric particles. For these analyses, B hadron tagging capability, coverage in the forward region and the overall hermeticity of the detector will be of fundamental importance. DELPHI plans to upgrade its vertex detector by extending the barrel by $24 \mathrm{~cm}$ and by adding a forward tracker consisting of two planes of silicon macropixel detectors and two planes of silicon ministrip detectors. The new detector is illustrated in figure 3. A description of the forward part of the tracker and the first test results can be found in [3]. In the barrel, the Closer layer, which already has a large angular coverage, will remain unchanged, and a new Inner layer will be built using double sided detectors from the present Outer layer and new single sided modules. Both the detectors and electronics of the upgraded Outer layer are new and were developed specifically for this project. In the next section the new layer and some first test results are described.

\subsection{The Outer Layer}

The Outer layer will contain 24 overlapping modules, each made up of two electrically independent ladders. Each ladder consists of 8 single sided detector plaquettes, placed back-to-back in two rows. The four plaquettes at smaller $\mathrm{R}$ have diodes parallel to the beam axis and give an $\mathrm{R} \phi$ measurement, and the four at greater $\mathrm{R}$ have diodes orthogonal to the beam axis and provide the $\mathrm{Rz}$ measurement. The plaquettes on each side of the ladder are daisy chained together and read out by a double sided hybrid. The same double metal technique described above for the Closer layer is used to read out the $\mathrm{Rz}$ signals. The plaquettes are separated from each other by a distance of about $0.6 \mathrm{~mm}$, the thickness of the hybrid, and held apart by ceramic spacers.

Figure 4 shows a schematic view of an Outer layer half module. The R $\phi$ plaquettes all have a strip pitch of $25 \mu \mathrm{m}$ with $50 \mu \mathrm{m}$ readout pitch. They are AC coupled, FOXFET biased detectors manufactured by Hamamatsu ${ }^{1}$. The Rz side detectors, manufactured by SINTEF $^{2}$, are also AC coupled but use polysilicon biasing. The strip pitch is a constant $50 \mu \mathrm{m}$ for each detector. However, as tracks coming from the interaction region will pass through the detector at widely varying angles relative to the readout coordinate, the readout pitch varies for the different plaquettes. The detector closest to the interaction point, labelled RZ1280, has two compartments with $44 \mu \mathrm{m}$ pitch and 640 readout strips. The next detector, labelled RZ640 has 640 readout strips and $88 \mu \mathrm{m}$ pitch, and the two outermost detectors have 320 readout strips each with $176 \mu \mathrm{m}$ pitch. There is therefore a four-fold multiplexing on each readout line. The readout chip used is the TRIPLEX [4], which was designed to withstand the high capacitative load of the long modules.

At present about half of the Outer layer modules have been constructed. The first measurements of the $\mathrm{S} / \mathrm{N}$ performance show values of 20 and 16 for the $R \phi$ and $R z$ side [5]. In the TRIPLEX chip it is possible to set the value of a variable resistor in front of the storage capacitors. In this way the relative amount of noise can be reduced and the $\mathrm{S} / \mathrm{N}$

\footnotetext{
${ }^{1}$ Hamamatsu Photonics K.K., Hamamatsu City, Japan.

${ }^{2}$ SINTEF, Oslo, Norway.
} 
can be improved by between $20 \%$ and $30 \%$, depending on the integration time allowed by the LEP beam configuration.

\subsubsection{Charge Loss on Intermediate Strips}

From the noise of the $\mathrm{Rz}$ detectors of the new Outer layer, it is possible to estimate a contribution to the capacitance from the second metal layer of about $1.2 \mathrm{pF} / \mathrm{cm}$, whereas the interstrip capacitance is about $1.4 \mathrm{pF} / \mathrm{cm}$. The intermediate strips therefore can couple to the second metal layer with a capacitance comparable to the strength with which they couple to their neighbours. When the charge is collected on the intermediate strip this can result in charge loss to the second metal layer. The extent of the charge loss was measured using a $904 \mathrm{~nm}$ GaAs laser to induce charge deposit in the detector. The single sided detectors have aluminised backing and therefore the laser must be shone onto the same side as the diodes. Note that the charge does not drift through the detector, and therefore this measurement does not analyse the depletion characteristics of the detector but simply the charge collection efficiency at any point. The laser is focussed onto the detector and moved in a direction perpendicular to the diodes in $0.5 \mu \mathrm{m}$ steps. In order to determine the exact position of the laser, the $\eta$ value at each point was simultaneously determined, where $\eta$ is defined as follows:

$$
\eta=\frac{\mathrm{PH}_{\max }}{\mathrm{PH}_{\text {left }}+\mathrm{PH}_{\text {right }}} .
$$

Here $\mathrm{PH}_{\max }$ is defined as the greater pulse height out of the two strip cluster made up of $\mathrm{PH}_{\text {left }}$ and $\mathrm{PH}_{\text {right }}$.

A value of $\eta$ close to 0.5 corresponds to a laser position between the readout lines and $\eta=1$ corresponds to a position directly above the readout lines. The measured S/N is shown in figure 5. Immediately above the diodes there is a reduction in the charge collected due to the aluminium read out line of width approximately $10 \mu \mathrm{m}$ which reflects the laser light. For the RZ320 detector, with no intermediate diodes, a regular structure can be seen with a loss of signal above the $44 \mu \mathrm{m}$ spaced readout lines (at $\eta=1$ ). From the width of the region with loss we estimate the width of the laser spot to be about $20 \mu \mathrm{m}$. The varying height of the peaks is due to small differences in the noise of the strips. For the RZ640 detector, there is an extra charge loss in addition to the loss on the aluminium readout lines. The loss is seen when the laser shines on the intermediate strips (at $\eta=0.5$ ) and corresponds to a loss of signal of about 50\%. For the RZ320 detectors, where there are two connected intermediate strips between two connected readout strips, a similar structure is seen, with a slightly greater charge loss of around $60 \%$. The charge loss was included in a full detector simulation in order to predict the expected loss of resolution. Averaging over all angles we find a degredation in resolution of between $10 \%$ and $14 \%$, depending on the noise cut used and the expected S/N. A degredation in efficiency is not expected.

\section{Conclusions}

The Closer layer double sided modules have been shown to operate at bias voltage which is below the regime at which 'micro-discharge' noise occurs. By tuning the bias voltage it is possible to achieve full efficiency on the ohmic side strips for the entire area of the 
plaquette. For the new 24cm long modules for the 1996 modules a $\mathrm{S} / \mathrm{N}$ of 25 (20) has been achieved for the $\mathrm{R} \phi(\mathrm{R} z)$ side. Due to the extra metal routing layer there is between $50 \%$ and $60 \%$ charge loss on the intermediate strips for three out of the four plaquettes. However the resolution is not expected to degrade by more than $15 \%$ due to this loss.

\section{Acknowledgements}

Many people have contributed to the measurements reported in this paper. In particular I have relied upon the work of Richard Brenner, Pawel Brückman, Hans Dijkstra, Yves Dufour, Carl Eklund, Michael Kaiser, Dieter Knoblauch and Agnieszka Zalewska. I am grateful to Guy Wilkinson for careful editing. 


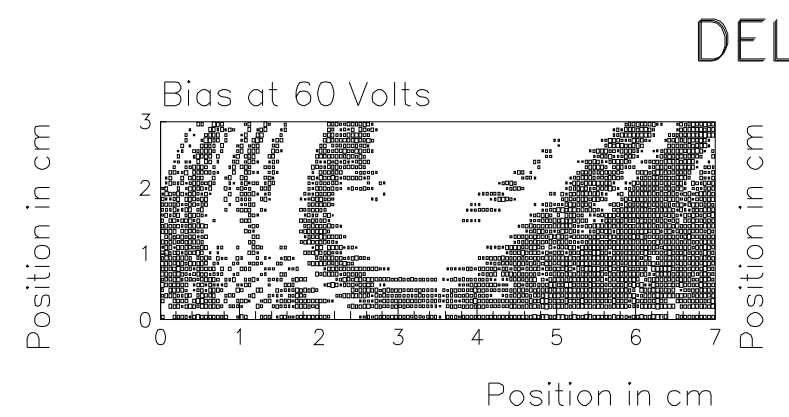

Bias at 75 Volts
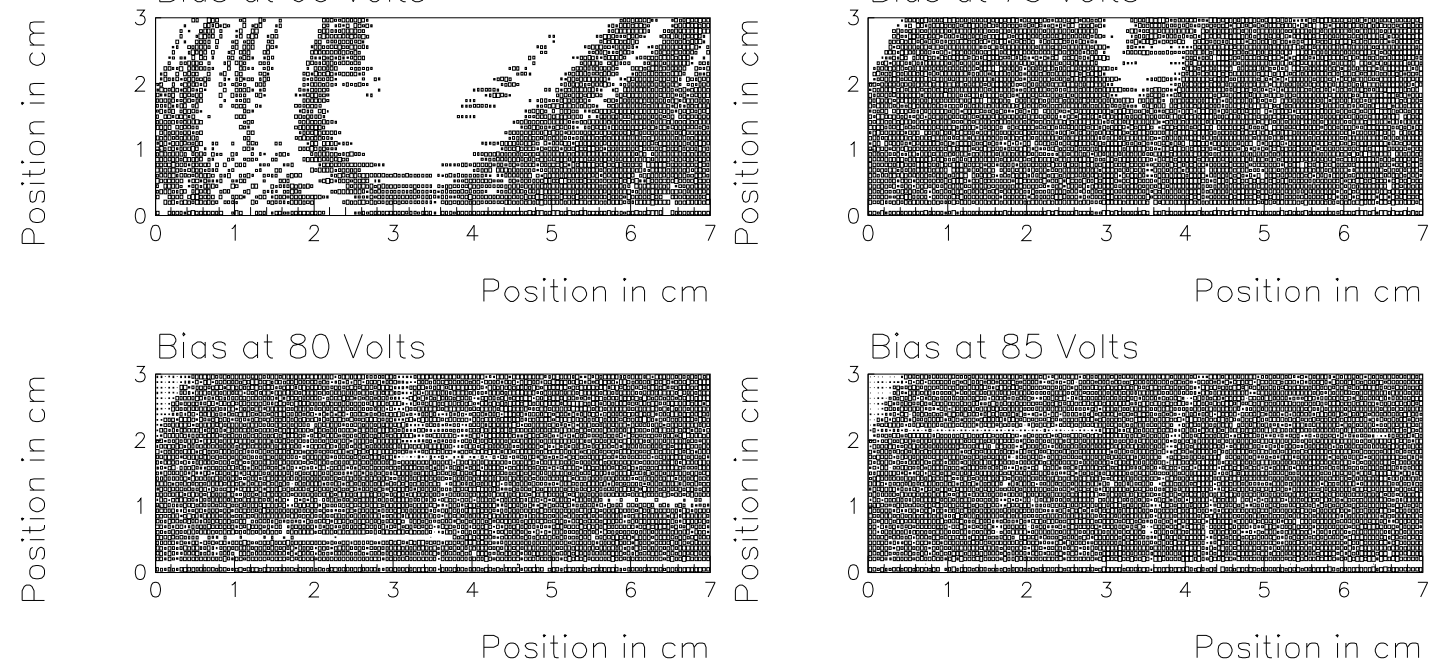

Figure 1: The signal measured at different points on the $n$ side of the Closer layer double sided $7.9 \mathrm{~cm}$ detector in response to a laser pulse shone on the $p$ side. The laser was moved in both directions in $500 \mu \mathrm{m}$ steps. The four plots show the response of the same detector at different depletion voltages. The empty regions indicate inefficiency in the detector. The horizontal and curved lines are artefacts of the measurement. 


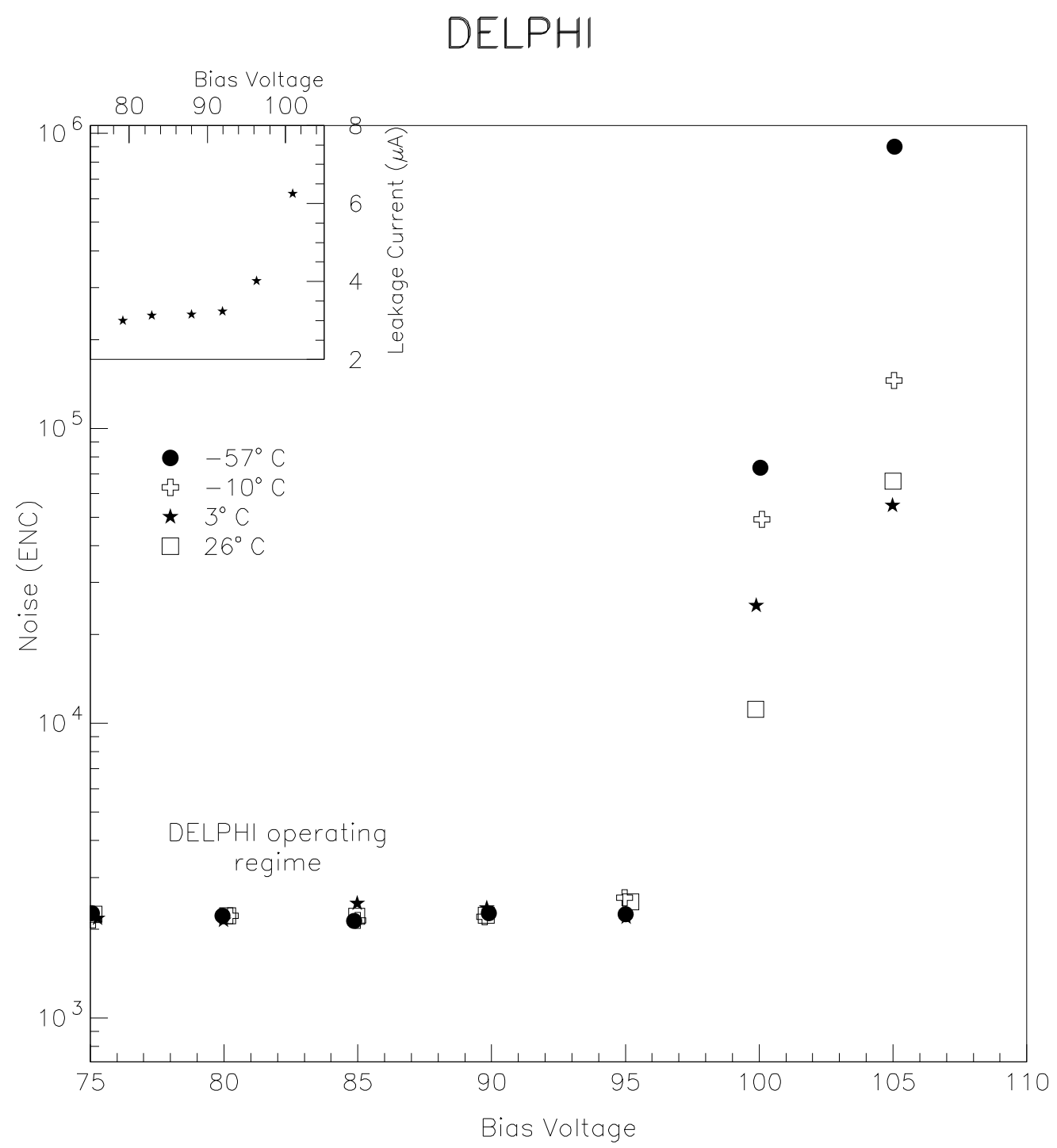

Figure 2: A measurement of micro-discharge noise in a DELPHI Closer layer detector for different temperatures. 


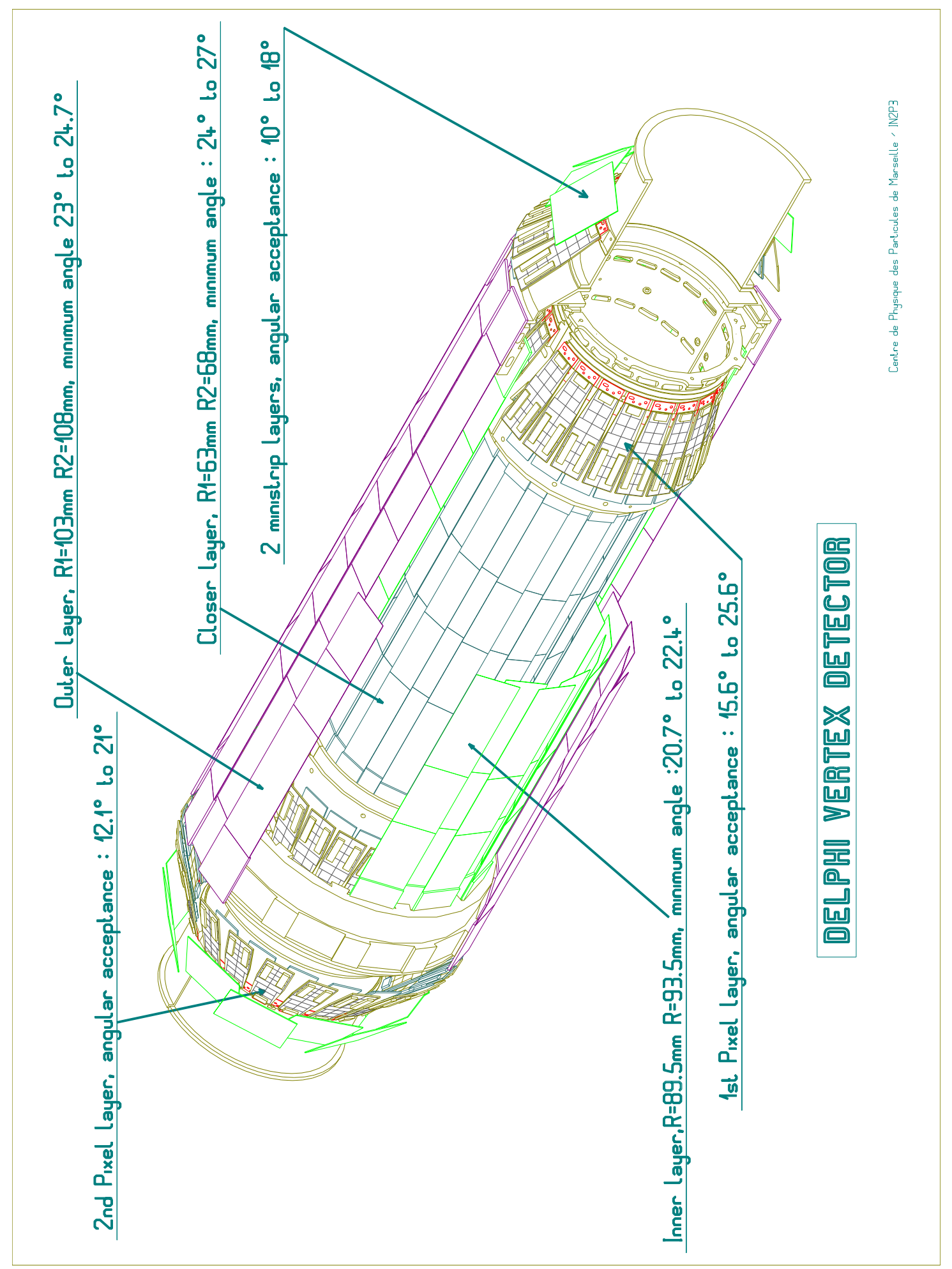

Figure 3: The 1996 DELPHI Silicon Tracker 


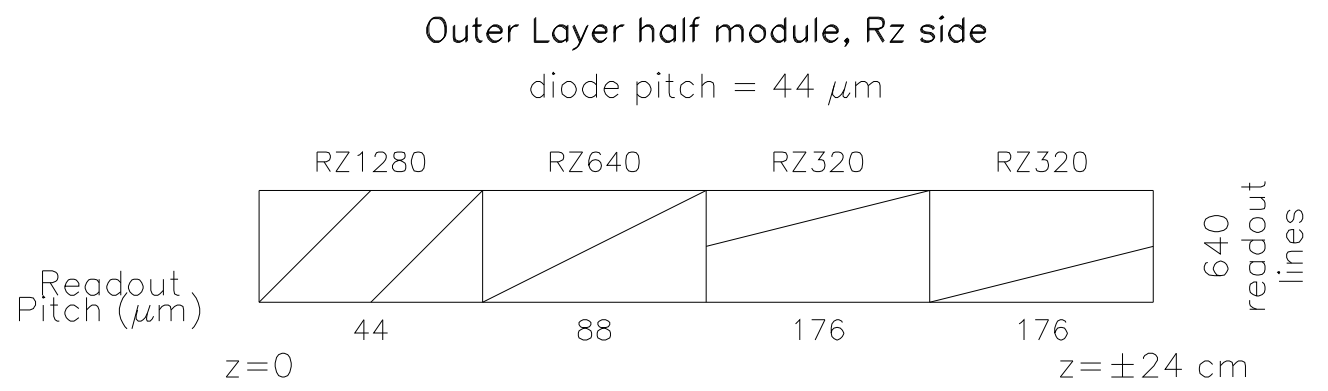

Figure 4: Multiplexing scheme for an Outer layer module. The diagonal lines indicate the lines of contact holes joining the first and second layers of metal lines. At the centre of DELPHI, where the tracks cross the plaquettes at an angle of $\theta=90^{\circ}$, the smallest pitch is used. 

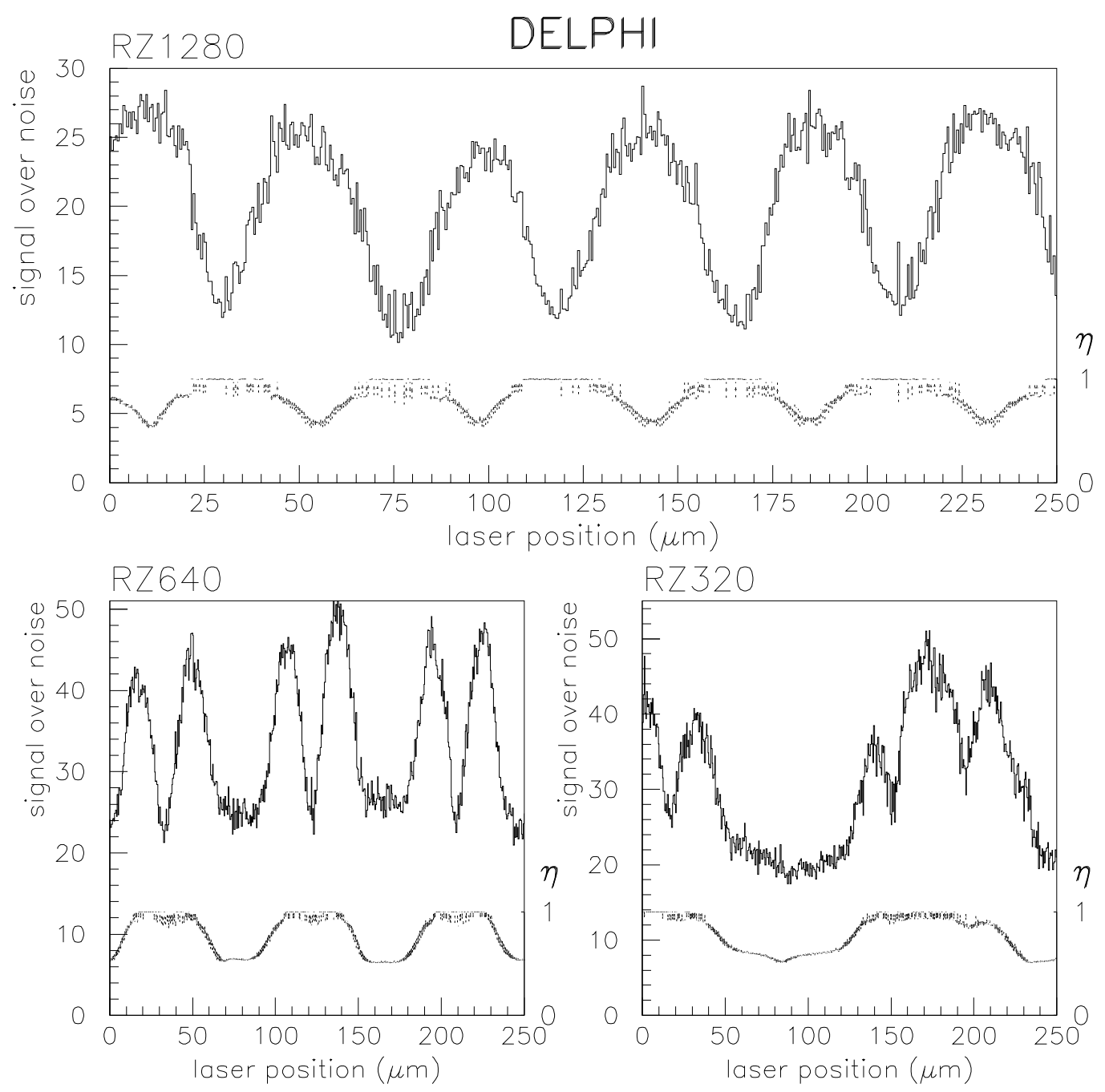

Figure 5: Measurement of the response of the three different kinds of detectors used in the new Outer layer to the laser pulse, as a function of the laser position. The left scale shows the signal over noise, which is the upper line, and the right scale indicates the value of $\eta$ at each point, which is the lower line. 


\section{References}

[1] V. Chabaud et al., "The DELPHI Silicon Strip Microvertex Detector with Double Sided Readout", CERN-PPE/95-86, 14 June, 1995.

[2] T. Ohsugi et. al., "Micro-discharges of AC-coupled silicon strip sensors", Nucl. Inst. Meth, A 342 (1994) 22-26.

[3] A. Andreazza et. al "The DELPHI Very Forward Tracker for LEP200", Proceedings of VII Wire Chamber Conference, Vienna, 13-17 Feb. 1995. To be published in Nucl. Instr. and Meth. A.

DELPHI Collaboration, "Proposal for the DELPHI Very Forward Tracker", CERN/LEPC/93-6 92-142.

[4] Ardelean et. al., Proceedings of the Nuclear Science Symposium, Oct. 30-Nov 1994, Norfolk, Virginia, USA, page 727.

[5] R. Brenner and C. Eklund, "First Testbench Measurements of the DELPHI Vertex Detector Outer Layer Modules for the 1996 Upgrade", HU-SEFT RD 1995-04. 